\title{
Ectopic Fgf signaling induces the intercalary response in developing chicken limb buds
}

\author{
Aki Makanae and Akira Satoh ${ }^{*}$ (D)
}

\begin{abstract}
Background: Intercalary pattern formation is an important regulatory step in amphibian limb regeneration. Amphibian limb regeneration is composed of multiple steps, including wounding, blastema formation, and intercalary pattern formation. Attempts have been made to transfer insights from regeneration-competent animals to regeneration-incompetent animalsat each step in the regeneration process. In the present study, we focused on the intercalary mechanism in chick limb buds. In amphibian limb regeneration, a proximodistal axis is organized as soon as a regenerating blastema is induced. Intermediate structures are subsequently induced (intercalated) between the established proximal and distal identities. Intercalary tissues are derived from proximal tissues. Fgf signaling mediates the intercalary response in amphibian limb regeneration.

Results: We attempted to transfer insights into intercalary regeneration from amphibian models to the chick limb bud. The zeugopodial part was dissected out, and the distal and proximal parts were conjunct at st. 24. Delivering ectopic Fgf2 + Fgf8 between the distal and proximal parts resulted in induction of zeugopodial elements. Examination of HoxA11 expression, apoptosis, and cell proliferation provides insights to compare with those in the intercalary mechanism of amphibian limb regeneration. Furthermore, the cellular contribution was investigated in both the chicken intercalary response and that of axolotl limb regeneration.

Conclusions: We developed new insights into cellular contribution in amphibian intercalary regeneration, and found consistency between axolotl and chicken intercalary responses. Our findings demonstrate that the same principal of limb regeneration functions between regeneration-competent and -incompetent animals. In this context, we propose the feasibility of the induction of the regeneration response in amniotes.
\end{abstract}

Keywords: Limb development, HoxA11, Intercalation, Limb regeneration, Fgf signaling, Chick, Axolotl

\section{Background}

The intercalary mechanism is considered a primary principle of regeneration in regenerative animals [1-5]. Intercalary pattern formation occurs following blastema formation. Blastema formation is considered to be the primary issue, since regeneration-incompetent animals, such as amniotes, do not form a blastema after damage. In blastema formation, the formation of an amputation plane is usually the start of blastema formation. The surrounding epidermis starts migrating to cover the exposed surface, forming wound epidermis/epithelium

* Correspondence: satoha@cc.okayama-u.ac.jp

Research Core for Interdisciplinary Sciences (RCIS), Okayama University, 3-1-1, Tsushimanaka, Kita-ku, Okayama 700-8530, Japan
(WE). Undifferentiated cells are accumulated beneath the WE, forming a regeneration blastema. Regeneration blastemas are thought to have a similar structure as developing limb buds. A similar or the same limb patterning mechanism as that for a developing limb bud appears to take place in order to remake a limb. The patterning mechanism in regeneration has been considered the intercalary pattern-forming mechanism. A regeneration blastema and an amputated plane initially re-establish a distal and a proximal positional identity, respectively [6-8]. Intermediate structures are then induced between the distal and the proximal tissues. This induction of an intermediate region by an established distal and an amputated stump is called "intercalation." These intercalary pattern-

(c) The Author(s). 2018 Open Access This article is distributed under the terms of the Creative Commons Attribution 4.0 International License (http://creativecommons.org/licenses/by/4.0/), which permits unrestricted use, distribution, and reproduction in any medium, provided you give appropriate credit to the original author(s) and the source, provide a link to the Creative Commons license, and indicate if changes were made. The Creative Commons Public Domain Dedication waiver (http://creativecommons.org/publicdomain/zero/1.0/) applies to the data made available in this article, unless otherwise stated. 
forming mechanisms in regeneration are conserved among species $[1,4]$.

Transferring the insights gained from regenerative animals to non-regenerative animals has been attempted. The first step, blastema formation, has been a significant challenge thus far, however as regeneration-incompetent animals cannot form a blastema. For example, mammalians cannot grow a blastema after limb amputation. Only the very distal tip retains regeneration potency. It is known that mice and even humans can regrow digit tips [9]. Generally, proximal amputation results in no regeneration response (no growth) in mice digits $[10,11]$. The application of Bmp proteins, however, can successfully induce the regeneration response [12]. However, the re-induction of a developmental field in a mature body has been a challenge in mice. The inability to induce a blastema after amputation can be also observed from the embryonic period in regenerationincompetent animals. When a developing chick limb bud is amputated, the amputated limb bud cannot regenerate the damaged region, and a truncated limb results [13, 14]. Even if an amputation is performed in the quite early stages, in which no skeletal elements are specified in a developing limb bud, no regeneration responses are observed. Interestingly, ectopic Fgf expression onto an amputated surface in the early stages can induce a distal structure $[13,15,16]$. Activation Fgf signaling induces regeneration responses, which appears to be a mechanism conserved from amphibians to chick embryos [17-19]. This induction of regeneration responses by exogenous Fgf signaling, however, can take place within the quite early developmental stages. In later stages, including in adult chicks, limb regeneration responses are no longer inducible by ectopic Fgfs [16]. Therefore, blastema induction remains a primary issue in regeneration-incompetent animals. This has prevented further study beyond blastema formation in regenerationincompetent animals.

Although the induction of a blastema continues to be a challenge, the use of chicken embryos allows us to investigate the latter phase, i.e. the intercalary pattern formation, in amniotes. Chicken embryos are easy to access if the eggshell is removed. Once an embryo is exposed, the limb buds are easy to manipulate. It is of note that chick embryos cannot regenerate amputated limb buds unless ectopic Fgf is applied to the amputated limb bud in the quite early stages $[13,15,16]$. Due to the easy accessibility of a chick embryo, it is possible to create a "pseudo-blastema" on an amputated limb bud. As mentioned above, a blastema is similar in structure to the distal part of a developing limb bud. When a chick limb bud is amputated, a distal part is always dissected out. In this study, we regarded an abscised distal part as a pseudo-blastema and placed it onto an amputated stump of a chick limb bud after dissecting out the expected zeugopodial region. This experimental design allows study of the intercalary processes that occur after blastema induction.

The present study focuses on the induction of the intercalary response in chick limb buds. Fgf signaling has been suggested to be involved in the process [7, 20-22]. In amphibians, when the intermediate structures were removed and a distal part (a hand part or a developing distal limb bud) was placed onto an amputated proximal structure, no intermediate regeneration could be observed. Ectopic Fgf application, however, resulted in the induction of intermediate structures in amphibians [21, 22]. This is thought to be regeneration of an intermediate structure. We aimed to investigate this intermediate regeneration in chick limb buds. Hamburger and Hamilton stage 24 (st. 24) chicken limb buds, in which most zeugopodial elements were already specified, were dissected into three pieces [23, 24]. The presumptive zeugopodial parts were removed, and the two pieces, the distal and proximal parts, were joined. We found that ectopic Fgf2 +8 application resulted in induction of the zeugopodial elements. Cell lineage tracing provides comparative insights to amphibian limb regeneration. Our findings suggest that induction regeneration responses in the limb buds of chick embryos are possible if discontinuity of the distal and proximal positional values is created under the presence of active Fgf ability. In other words, the primary principal of limb regeneration, the intercalary response, can be induced in a regeneration-incompetent chick embryo.

\section{Methods \\ Experimental manipulations}

Chicken embryos were staged according to the methods of Hamburger and Hamilton [23]. Stage 24 limb buds were amputated at $250 \mu \mathrm{m}$ and $800 \mu \mathrm{m}$ from the distal tip with forceps. Distal fragments of the dissected tissues were grafted onto proximal stamps with Fgf2 +8 -soaked or PBS-soaked beads by tungsten needles. The beads were prepared following previously described methods [25]. To visualize the skeletal pattern, the embryos were incubated for 7 days after surgery and stained with Alcian blue.

Axolotls (Ambystoma mexicanum) with a nose-to-tail length of 8-12 cm were obtained from private breeders and housed in aerated water at $22{ }^{\circ} \mathrm{C}$. Green fluorescent protein (GFP) transgenic axolotls were obtained from the Ambystoma Genetic Stock Center (AGSC). Hand grafting procedures followed the previous report [21]. 


\section{Sectioning and histological staining}

Samples were fixed with $4 \%$ paraformaldehyde for 1 day at room temperature. If necessary, decalcification by $10 \%$ EDTA was performed for 1 day. Samples were embedded in O.C.T. compound (Sakura Finetek, Tokyo) following 30\% sucrose/phosphate-buffered saline (PBS) treatment for approximately $12 \mathrm{~h}$. Frozen sections of $14 \mu \mathrm{m}$ thickness were prepared using a Leica CM1850. The sections were dried thoroughly under an air dryer and kept at $-80{ }^{\circ} \mathrm{C}$ until use.

Standard haematoxylin and eosin (HE) staining was used for histology. To visualize cartilage formation, Alcian blue staining was performed before HE staining. In brief, sections were washed in tap water several times to remove the O.C.T. compound. Then, Alcian blue (Wako, pH 2.0) solution was dropped on the section, and the slide was incubated for $5 \mathrm{~min}$. The sections were washed twice with tap water, and then HE staining was performed. The stained sections were mounted using Softmount (Wako, Osaka). For whole-mount skeletal staining, we used the procedures reported by [21].

\section{In situ hybridization, immunofluorescence, and in situ apoptosis detection}

The following genes were cloned by reverse transcription polymerase chain reaction (RT-PCR): $P g-h$, Meis2, Shox2, Hoxa-11, and Hoxa-13. RNA probe templates were synthesized by PCR using M13 forward and reverse primers. Based on sequence data, the appropriate RNA polymerase was selected to synthesize antisense RNA probes. A labelled Hoxa-11 probe was subjected to alkaline hydrolysis to obtain optimal signals. Whole-mount and section in situ hybridization for chick embryos was performed using standard methods. Gdf5 and type II collagen probe for in situ hybridization for amphibians were described in the previous study [26, 27]. Immunofluorescence on sections was carried out on the basis of previous reports [28]. Chicken cell marker antibody (8F3, 1:200) and quail cell marker antibody (QCPN, 1: 100) were obtained from the Developmental Studies Hybridoma Bank (DSHB). Anti-phospho-histone H3 antibody (1:500) was purchased from Cell Signaling Technology. Anti-GFP (1:500) was purchased from Clontech. Anti-mouse IgG Alexa 488 (1:500) and antirabbit IgG Alexa 488 (1:500) were purchased from Invitrogen. Nuclei were visualized by Hoechst 33,342 (Dojindo) staining. Images were captured using an Olympus BX51 system. All experiments were performed three times to confirm the results. Statistical analysis was performed by t-test (one-tail). Cell counting was performed in three independent samples.

Apoptotic cells were detected with an In Situ Apoptosis Detection Kit (TaKaRa Bio Inc.). Staining was performed according to the kit manual. Sections were washed in PBS and covered with permeabilization buffer for 5 min on ice. After the PBS wash, TdT labelling solution was dropped onto the slides before incubation at $37{ }^{\circ} \mathrm{C}$ for $90 \mathrm{~min}$. The labelled slides were washed in PBS containing Hoechst 33,342 (Dojindo) and mounted coverslips. All experiments were performed three times to confirm the results. Statistical analysis was t-test. Cell counting was performed from three independent samples.

\section{Results}

\section{Induction of intercalary responses in st. 24 chick limb buds}

The experimental design is illustrated in Fig. 1a-e. The experimental procedures are summarized in Fig. 1b. St. 24 chick embryos were used. Zeugopodial cartilaginous elements (radius/ulna) were targeted and dissected out, as shown in Fig. 1c-e. The distal part was placed onto the amputated stump, and an Fgf2 +8-soaked or phosphate-buffered saline (PBS)-soaked bead was settled between the distal part and the proximal part (Fig. 1e). This surgery joined the distal part to the amputated stump, which is similar to when an early blastema forms on an amputated stylopod, since an early blastema possesses a distal identity. For the purpose of prospecting the st. 24 limb bud, in situ hybridization was performed (Fig. 1f-m). Limb buds at st. 24 already specified the zeugopodial elements (Fig. 1f). Cartilage-specific proteoglycan $(P g-h)$ expression visualizes the ulna elements although the radius continued to have no distinguishable PG-H expression ([29]; Fig. 1f). Stylopod cartilage is usually determined at this stage. Pg- $h$ expression, however, could not be detected by whole-mount in situ hybridization, due to the thickness of the limb bud (a technical reason). The grafting procedure eliminated the zeugopodial elements. Therefore, the $P g$ - $h$ expression domain in the zeugopod disappeared completely (Fig. 1j). Meis2 and Shox2 are the proximal marker genes, which mainly express in a stylopodium region ([30, 31]; Fig. 1g, h). These gene expressions could not be detected in the distal graft, indicating that there was no proximal cell contamination in the distal graft (Fig. 1k, l). HoxA13 is an autopod marker gene (Fig. 1i). HoxA13 was expressed in the grafted distal part but not in the proximal stump at all (Fig. 1m). These results support our choice of experimental design.

The skeletal pattern caused by the surgery was investigated. Chick embryos were harvested on day 11 of incubation. The skeletal pattern was visualized by Alcian blue staining. The skeletal pattern of an intact (non-amputated) chick limb is shown in Fig. 2b. Limb bud simply amputated one-quarter from the proximal border caused severe loss of distal parts (Fig. 2c). Importantly, the distal half of the stylopod was always absent and no zeugopodial elements were 

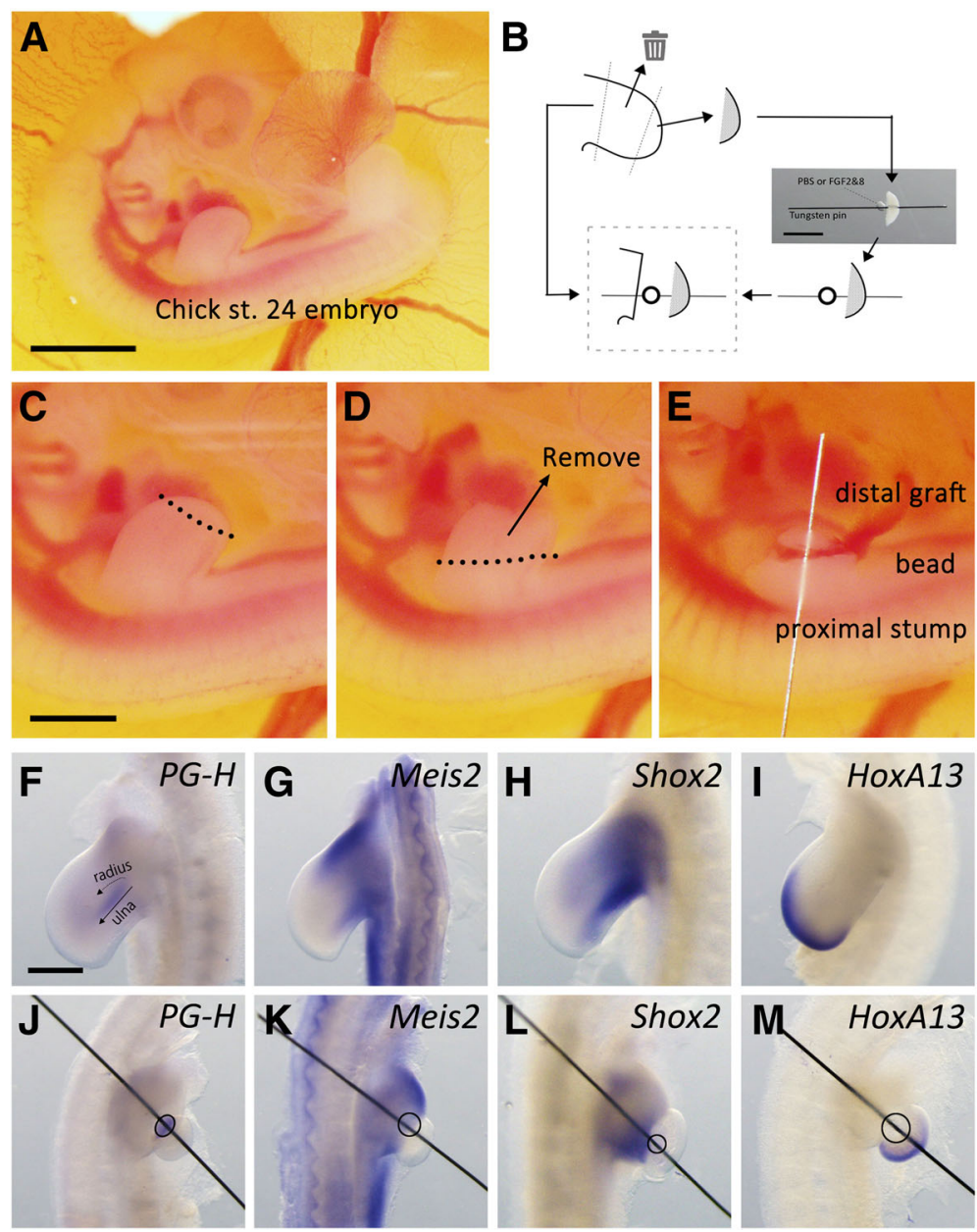

Fig. 1 Summary of the experimental procedures and gene expression pattern. a Chicken embryo at stage 24, which was used for the surgery in the present study. b-e Summary of surgical procedures. b The schematic diagram of the surgical procedure. The distal one-quarter was isolated and stabbed with a gelatin bead. c The distal one-quarter of the limb bud was dissected out and the isolated part was used for the graft. $\mathbf{d}$ The next two quarters, the intermediate part, was removed. e The remaining one-quarter of the stump was stuck with the distal graft and the bead. $\mathbf{f}-\mathbf{m}$ Gene expression pattern in non-amputated limb buds $(\mathbf{f}-\mathbf{i})$ and operated limb buds $(\mathbf{j}-\mathbf{m})$. Pg-h (Aggrecan) expression ( $\mathbf{f}, \mathbf{j})$, Meis2 expression $(\mathbf{g}, \mathbf{k})$, Shox 2 expression $(\mathbf{h}, \mathbf{I})$, and HoxA13 expression (i, $\mathbf{m})$. The circles indicate the position of the grafted bead (J-M). The scale bars in $\mathbf{a}, \mathbf{b}, \mathbf{c}$, and $\mathrm{F}$ are $2,1,1$, and $0.5 \mathrm{~mm}$, respectively

ever observed. When one-quarter of a distal part and one-quarter of a proximal part were joined and a PBS-soaked bead was placed between them, the zeugopod elements were not reformed. The autopod elements were directly elongated from the stylopod element (Fig. 2d, Table 1). When the Fgf2 + Fgf8soaked bead was placed on the border of the graft, the zeugopod elements were induced (Fig. 2e, Table 1, Additional file 1: Figure S1). Some exhibited relatively normal radius and ulna cartilage $(n=9 / 21 ; 43 \%)$, and some exhibited truncated but identifiable zeugopodial elements $(n=7 / 21 ; 43 \%$, Additional file 1: Figure S1). This suggests that ectopic Fgf signaling mediates the intercalary response in chick limb buds.

\section{HoxA11 expression, cell death, and proliferation in the intercalary reaction}

To characterize the induced intermediate structure by Fgf2 + Fgf8 application, a molecular marker gene of a zeugopod was necessary. HoxA11 has been used as a typical zeugopodial marker gene in amniotes. HoxA11 is first expressed from the very distal tip of a limb bud, including to the presumptive autopodial region. Later, HoxA11 expression starts being suppressed in a distal tip of a limb bud around st. 25 [32], and the HoxA11 expression domain is relatively restricted in the zeugopodial domain [32-34]. A knockout of the Hox11 paralogous group (Hoxa11, Hoxc11, and Hoxd11) results in severe underdevelopment of the zeugopodial 


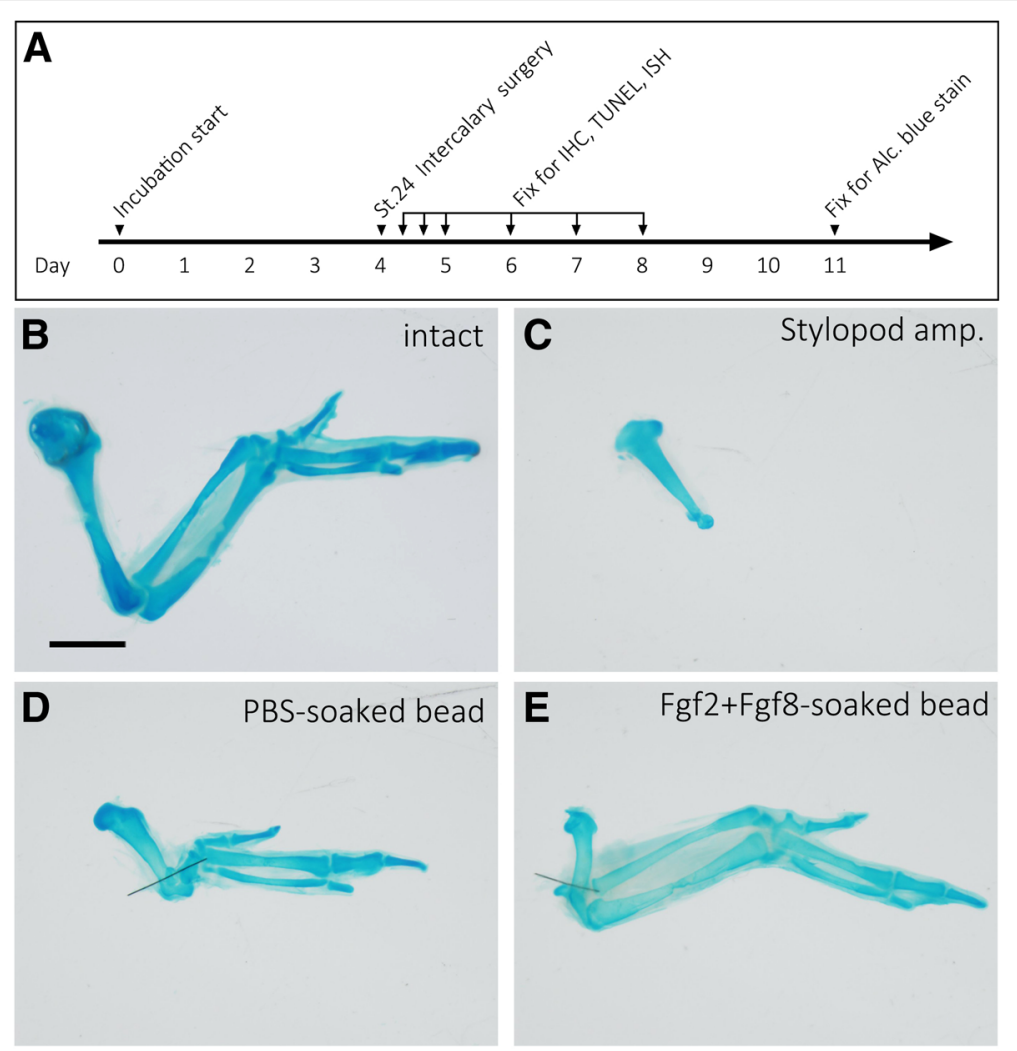

Fig. 2 The skeletal phenotypes. a Time schedule of the experiment. b The intact chicken wing. c The skeletal pattern derived from the amputated limb bud three-quarters from the distal tip at st. 24. $\mathbf{d}$ The skeletal pattern of the limb bud from which the middle part was dissected out. An intercalary induced structure could be observed. e An Fgf2 + Fgf8-soaked bead induced the intermediate (zeugopodial) structures between the stump and the graft. The scale bar in B is $2 \mathrm{~mm}$

elements, and relatively normal autopod development [35]. These findings indicate that HoxA11 is a reasonable zeugopodial marker gene.

The skeletal pattern strongly suggested that the zeugopodial elements were induced by ectopic Fgf2 + Fgf8 application (Fig. 2e). To confirm this further, HoxA11 expression was investigated in the operated limb buds (Fig. 3). Removal of the presumptive zeugopodial part and bead grafting were performed. Samples for in situ hybridization were collected $2-4$ days after surgery. In the control, HoxA11 expression was detectable in the proximal region of the grafted distal part (Fig. 3a). On day 7 (3 days after surgery), HoxA11 expression disappeared and became unrecognizable (Fig. 3c). In later periods, no $\operatorname{HoxA11}$ was detectable (Fig. 3e, data not shown). In contrast, HoxA11 expression was maintained in the Fgf2 + Fgf8 bead-grafted limb (Fig. 3b, d, f). On day 6 ( 2 days after surgery), HoxA1lexpression was identifiable on the control side (Fig. 3a, b). Notably, HoxA11 remained detectable within the proximal region in the later stages (Fig. 3d, f). The HoxA11 expression domain appeared to be induced within the stump region. This result indicates that HoxA11 expression was induced under the condition of ectopic Fgf signaling and discontinuity of the PD positional values.

Cell proliferation and apoptosis were also investigated in the intercalary response (Figs. 4, 5). Mitogenic activity was measured by phosphorylated histone $\mathrm{H} 3(\mathrm{pH} 3)$, which is used as a specific marker of S-phase. The $\mathrm{pH} 3$ positive cells were counted from three independent samples at each time point (Fig. $4 \mathrm{~m}$ ). Cell counting was performed around the place where bead was observed. Unexpectedly, no apparent difference between the control and the Fgf-grafted limb was observed at any time point

Table 1 Fgf-mediated intercalary responses in st. 24 chicken limb buds

\begin{tabular}{lllll}
\hline & Zeugopod induced & Intermedial cartilage formed & No zeugopod & Total \\
\hline FGF2 + FGF8 & 9 & 7 & 5 & 21 \\
PBS & 0 & 2 & 18 & 20 \\
\hline
\end{tabular}




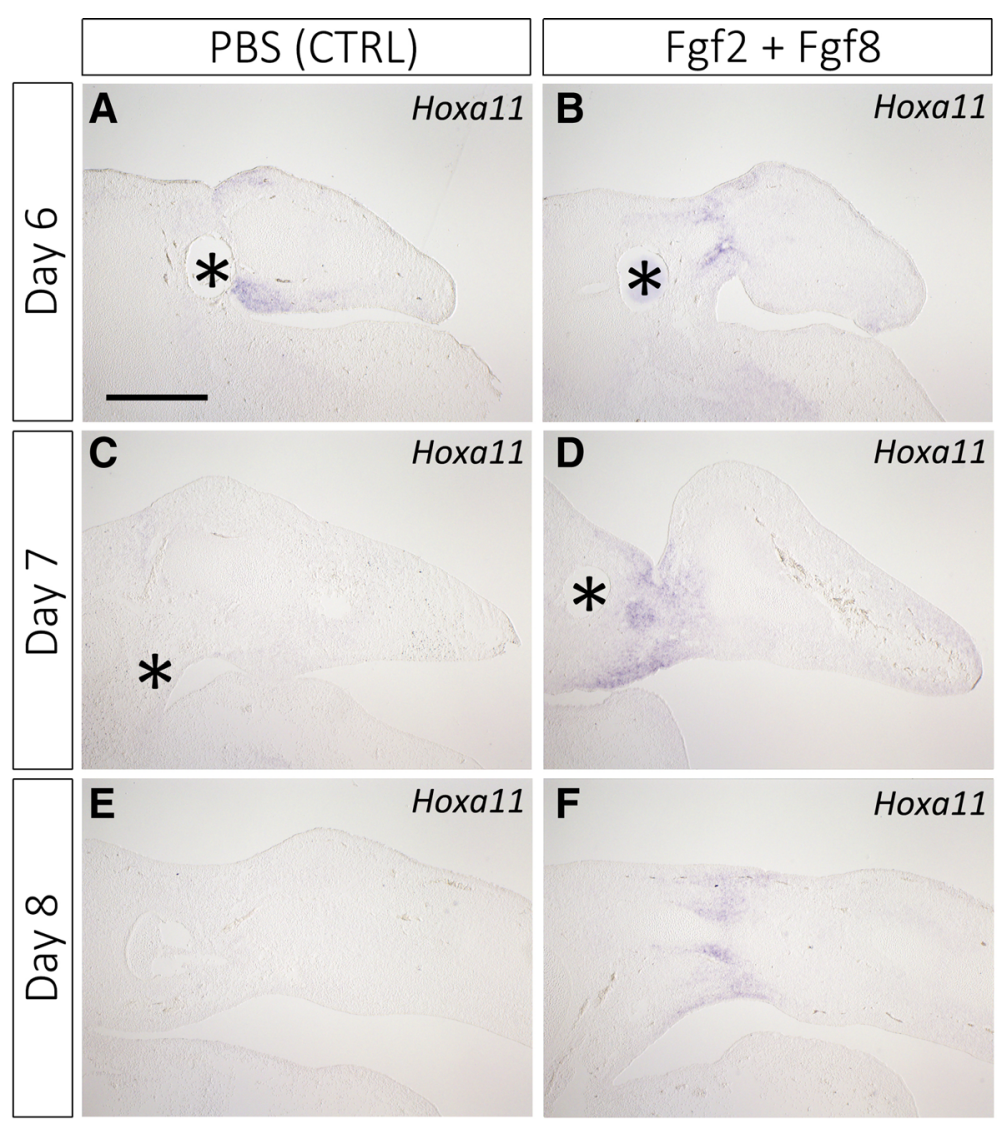

Fig. 3 HoxA11 expression pattern in the operated limbs. HoxA11 expression in the control (PBS) samples (a, c, e) and Fgf2 + Fgf8 bead-grafted limb buds (b, d, f). a, b HoxA11 expression remained at the distal parts on day 6 (2 days after surgery). HoxA11 expression disappeared in the control samples at later time points, however $(\mathbf{c}, \mathbf{e})$. $\mathbf{d}, \mathbf{f}$ HoxA11 expression was observed in the proximal region in the Fgf2 + Fgf8 bead-grafted limbs. Asterisks indicate the grafted beads. The scale bar in A is $500 \mu \mathrm{m}$

(Fig. $4 \mathrm{~m})$. At the early time points ( 8 and $16 \mathrm{~h}$ ), however, the ischemic region, where $\mathrm{pH} 3$ positive cells were not detected, was sometimes observed (Fig. 4a-d, arrowheads). Apoptotic cells were visualized by TUNEL analysis (Fig. 5). Apoptotic cells became apparent within $16 \mathrm{~h}$ (Fig. 5a-d, m). Fgf 2 + Fgf8 application appeared to suppress apoptosis compared to the control (Fig. 5a-d, m). At later time points, few apoptotic cells could be detected in either sample (Fig. 5e-1). These results suggest that neither cell proliferation nor cell death is the primary force of the intercalation response in chick limb buds.

\section{Lineage tracing analysis reveals proximal dominant contribution}

Intercalation in limb regeneration processes has mainly been studied in amphibians. Classic cell lineage analysis revealed that stump tissues contribute to newly induced intermediate structures [36-38]. When a distal (autopodial) part was placed onto an amputated stylopod, the regenerated intermediate (zeugopod) structures consist of stylopod-derived cells. This finding has not, however, been re-evaluated using recent molecular technology. Recently, Fgf-mediated intercalation was demonstrated in axolotl limbs $[7,21]$. The hand grafting technique in a mature axolotl was first pioneered by Bryant and Item [39]. The hand part was dissected out and placed onto an amputated stylopod. Essentially, no zeugopodial elements were regenerated ([21]; Fig. 6e). A previous study by our group demonstrated that Fgf 2 application between the hand part and the amputated stylopod could induce zeugopodial elements [21]. We first re-examined this using a GFP transgenic and a normal axolotl. The summary of the experimental design is illustrated in Fig. 6a. The hand part was derived from a wild-type axolotl and the host was a GFP transgenic animal. Fgf2 + Fgf8 was used in order to achieve similar experimental conditions to those of the chick experiments. As reported previously, ectopic Fgf application to the border of the graft successfully induced zeugopodial elements $(n=6 / 10$; [21]; Fig. 6b-d). No obvious morphological change was observed 10 days after Fgf2 + Fgf8 application (Fig. 6b). A recognizable extension was observed between the stump and the grafted hand part 30 days after the 


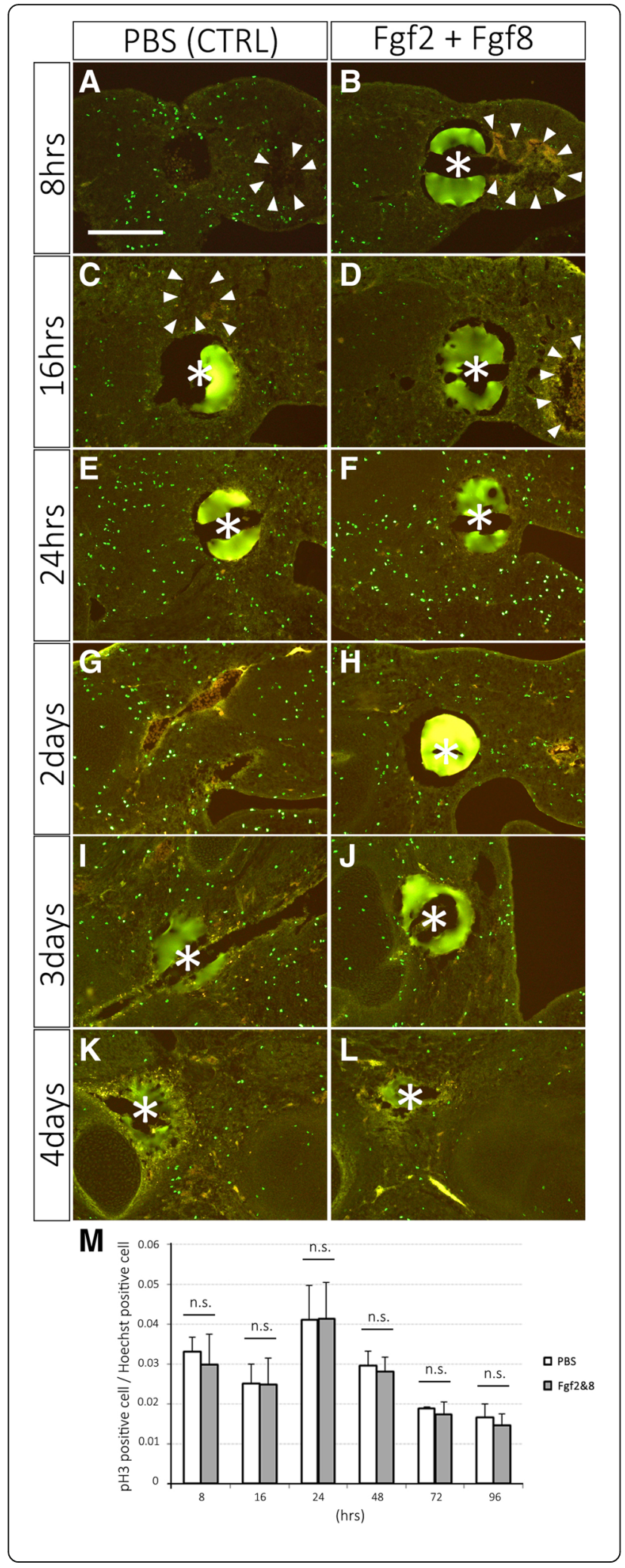

Fig. 4 Mitogenic activity was measured by $\mathrm{pH} 3$ immunofluorescence. The left column shows the control samples with the PBS-soaked bead. The right column shows the limb buds with the Fgf2 + Fgf8-soaked bead. Asterisks and arrowheads indicate the beads and the ischemic region, respectively. The scale bar in A is $250 \mu \mathrm{m}$. (M) The summary of the result of $A-L$ and the statistical analysis. n.s. $=$ no significant difference

surgery (Fig. 6c). It was difficult to observe for much longer than this because of immuno-rejection of the graft. Alcian blue staining revealed the skeletal pattern (Fig. 6d, e). In the Fgf2 + Fgf8-grafted samples, the induced zeugopodial elements could be observed (Fig. $\left.6 \mathrm{~d}, \mathrm{D}^{\prime}\right)$. In contrast, the PBS-soaked bead grafting resulted in no regeneration of zeugopodial elements (Fig. 6e, E'). To investigate the cellular contribution, the Fgf2 + Fgf8 bead-grafted samples were fixed and sectioned (Fig. 6f). Consistently, the histological analysis revealed that the zeugopodial elements were induced by Fgf 2 + Fgf8 application (Fig. 6f). The induced cartilaginous elements existed independently from the autopodial and stylopodial skeletal elements (Fig. 6f). The border cells of the induced zeugopod cartilage expressed Gdf5 (Additional file 2: Figure S2). This suggests that each element was segregated by jointlike formation. Cartilaginous cells were visualized by type II collagen (Col2A) in situ hybridization, and GFP-positive cells were visualized by immunofluorescence (Fig. 6g-s). For the induced zeugopod, the distal region consisted of two populations, GFP-positive and GFP-negative (Fig. 6j, k, m, n). In contrast, the proximal region of the induced zeugopod was composed of host-derived cells (GFP-positive; Fig. 61, o). This result was further confirmed in animals that had a GFP-positive graft and a GFP-negative host (Fig. 6p-s). In these animals, the induced zeugopodial elements consist of GFP-positive cells in the distal part and GFP-negative cells in the proximal part (Fig. 6p-s). These results clearly demand renewal of the classic understanding of the intercalary response in amphibian limb regeneration. Consistently, most of the intercalary induced tissues were derived from the proximal tissues. However, distal tissues can also contribute to the intercalary induced tissues in the distal region.

As shown above, the classical understanding of the cellular contribution of intercalary regenerated tissue needs to be revised. Based on the results shown in Fig. 6, the cellular contribution of chick intercalary induced tissues was investigated (Fig. 7). Quail-chick chimeras have been used for cell lineage tracing experiments in chick developmental studies. A distal limb bud was obtained from a quail limb bud, and a chicken limb bud was used as a host. Samples were fixed on day 7 after surgery. Histological observation revealed that Fgf application induced the cartilage 


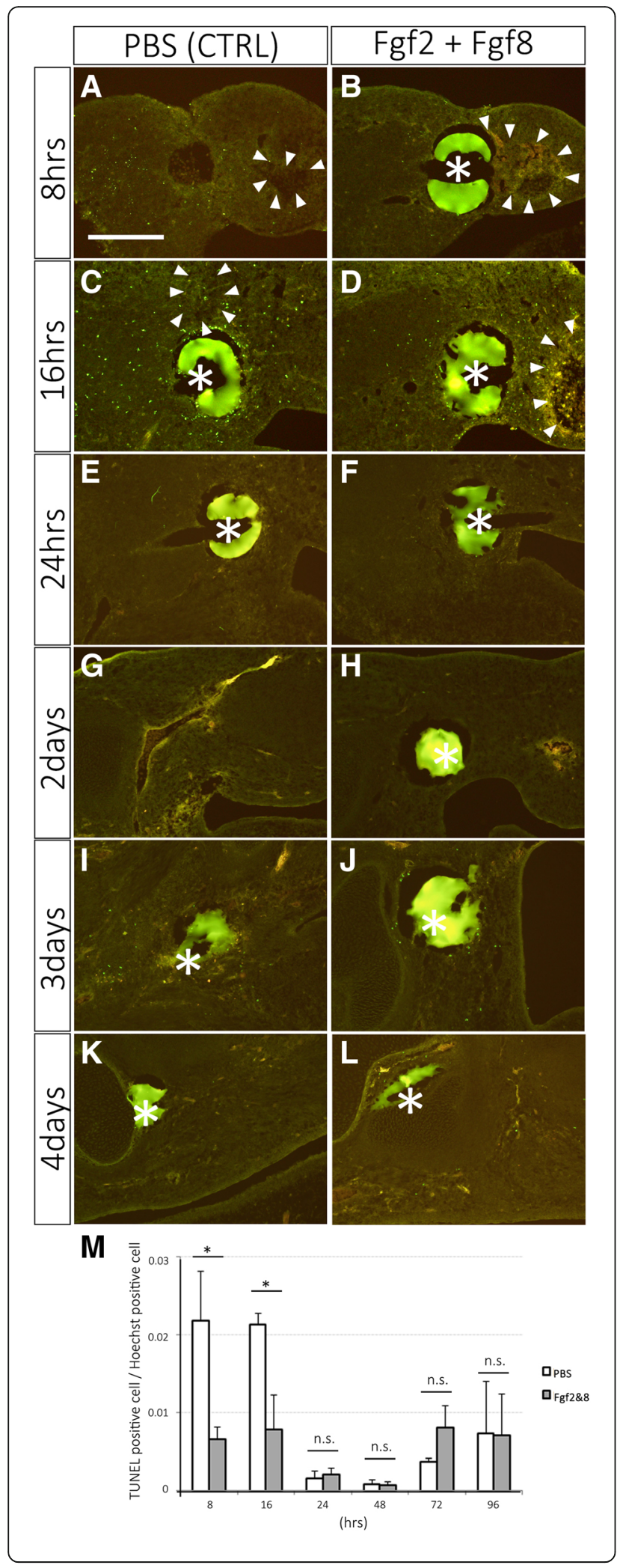

Fig. 5 Apoptosis was investigated by TUNEL analysis. The left column shows the control samples with the PBS-soaked bead. The right column shows the limb buds with the Fgf2 + Fgf8-soaked bead. Asterisks and arrowheads indicate the beads and the ischemic region, respectively. The scale bar in $A$ is $250 \mu \mathrm{m}$. (M) The summary of the result of $A-L$ and the statistical analysis. n.s. $=$ no significant difference. ${ }^{*}=P<0.05$

structure between the distal graft and the stump whereas the PBS-soaked bead grafting did not give rise to any ectopic structures (Fig. 7a, b). To determine the cell contribution, chick-specific antigen (8F3) was used in the immunofluorescence (Fig. 7c, d). In the control, a clear boundary could be observed (Fig. 7c, C'). There was no cartilage consisting of both chick-derived and quail-derived cells (Fig. 7c, C'). It was clear that the host cells penetrated into the distal region (Fig. 7c, d). In limb development, migrating cells, such as muscle cells, endothelial cells, fibroblasts, and neural cells, toward a distal region exist, which accounts for the 8F3-positivecells in the distal region (Fig. 7c, d). The Fgf2 + Fgf8-induced cartilage constituted of the two populations (Fig. 7d). Within the stylopod and the autopod region, cartilage consisted of either population (Fig. 7d). It was evident that two populations contributed to the intercalary induced cartilage (Fig. 7d'). Within the induced cartilage, most of the cartilage cells were derived from the host (8F3-positive). The distal region of the induced cartilage was derived from the graft (8F3-negative; Fig. 7D'). QCPN immunofluorescence was conducted at the location of the graft (quail) cells (Fig. 7e, f). The results were perfectly consistent with $8 \mathrm{~F} 3$ immunofluorescence. The cellular contribution was similar to that of intercalary regeneration in an axolotl (Fig. 6). This result suggests that intercalation in chick limb buds is regulated in a similar manner as that of urodele amphibians.

We also investigated the relationship between HoxA11 re-expression and cellular contribution. In this experiment, the host was derived from a chick embryo and the graft was from a quail limb bud. Samples were fixed 4 days after the surgery. HoxA11 expression was determined by in situ hybridization (Fig. $7 \mathrm{~g}$ ), and chicken cells were clarified by $8 \mathrm{~F} 3$ immunofluorescence on the adjacent section (Fig. 7H). The HoxA11 expression domain appeared to be within the 8F3-positive domain (Fig. $7 \mathrm{~g}-\mathrm{i}$ ). This suggests that the proximal cell population mainly generates HoxA11-positive zeugopodial elements during the intercalary response.

\section{Discussion}

\section{Intercalary regulation in chick limbs}

Intercalary regulation is quite common in amphibian limb regeneration. Bryant and her colleagues first proposed that distal and proximal interaction creates 


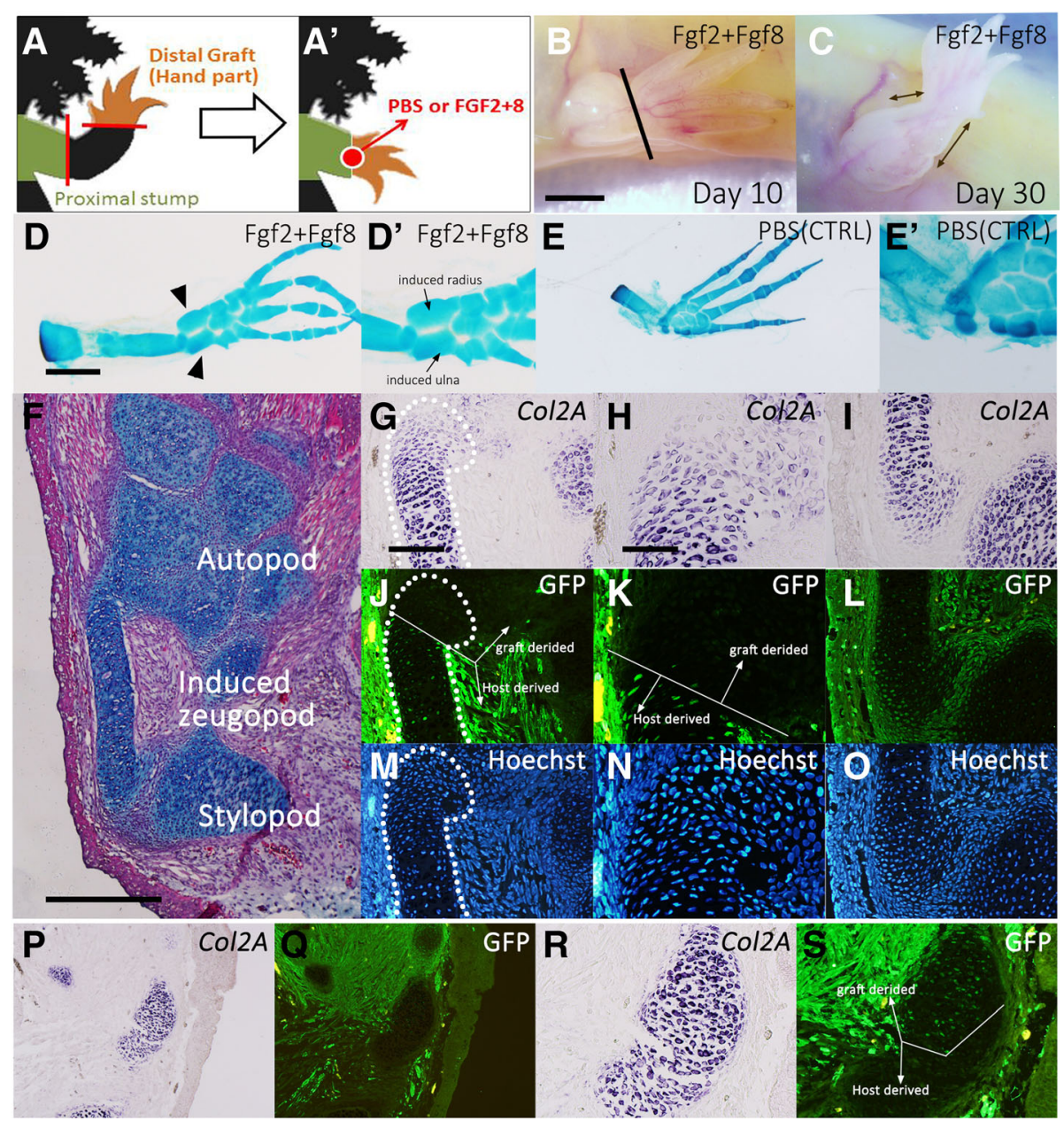

Fig. 6 Re-evaluation of cellular contribution in the axolotl intercalary response. a Schematic diagram of the experimental design. $\mathbf{b}$ Hand grafting 10 days after surgery. The line indicates the approximate border of the graft. The scale bar is $3 \mathrm{~mm}$. c Day 30. The elongated portion is recognizable between the grafted hand and the stump (double-headed arrows). d, e Skeletal pattern was revealed by Alcian blue staining. $\mathbf{d}$ Fgf2 + Fgf8 bead-grafted sample. Arrowheads indicate the two induced cartilages between the graft and the stump. (D') Higher magnification view of D. e PBS-soaked bead grafting resulted in no intercalary responses (E'). $\mathbf{f}$ Histological observation of the Fgf2 + Fgf8 bead-grafted limb. Cartilage formation is readily evident between the hand graft and the stump. The scale bar indicates $1 \mathrm{~mm}$. $\mathbf{g}$-o The identical section underwent in situ hybridization and immunofluorescence. (G-I) Type // collagen (Co/2A) expression pattern in the intercalary induced cartilage. (J-L) GFP expression pattern in the intercalary induced cartilage. The host was a GFP transgenic axolotl. Proximally derived cells were GFP-positive. $\mathbf{m}-\mathbf{o}$ Hoechst staining for nuclei. $\mathbf{g}, \mathbf{j}$, and $\mathbf{m}$ are the distal regions of the induced zeugopod, and $\mathbf{i}, \mathbf{I}$, and $\mathbf{o}$ are the proximal. $\mathbf{H}, \mathrm{K}$, and $\mathbf{N}$ are the higher magnification views of $\mathbf{g}$, j, and $\mathbf{m}$, respectively. The scale bars in $\mathrm{G}$ and $\mathrm{H}$ are 500 and $200 \mu \mathrm{m}$, respectively. $\mathbf{p}-\mathbf{s}$ The same experiment was performed with a normal host animal and a graft derived from a GFP transgenic axolotl. p, $\mathbf{r}$ ) Type I/ collagen (Col2A) expression pattern in the intercalary induced cartilage. $\mathbf{q}, \mathbf{s}$ GFP expression pattern in the intercalary induced cartilage. $\mathrm{R}$ and $\mathrm{S}$ are higher magnification views

intermediate structures [4]. This concept has not, however, been applied to chick limbs [40]. A classical grafting experiment reported that intercalation did not take place if a distal part was transplanted onto an amputated limb bud at an expected stylopod [14]. It is of note that the induction of intermediate structures in this grafting experiment was reported in chick limb buds in the early stage [41, 42]. Very early chicken limb buds, especially around st. 20, do not have a distinguished zeugopodspecified population [43, 44]. The very distal tip of an early-stage limb bud contains expected autopods and zeugopods. HoxA13, an autopod marker gene, is upregulated from st. 21 [32, 45]. Therefore, it is not surprising that the distal graft contains zeugopodial parts in these early experiments. As shown in Fig. 1, a limb bud at st. 24 has distinguished autopod, zeugopod, and stylopod regions. Furthermore, zeugopodial cartilaginous elements were already specified, and recognized as $P g-h$ positive radius/ulna cartilage (Fig. 1f). The use of st. 24 limb buds decreased the chance to contaminate any zeugopodially specified cells in the grafted distal part (Fig. 1b). Nevertheless, it remains possible that a distal part was 

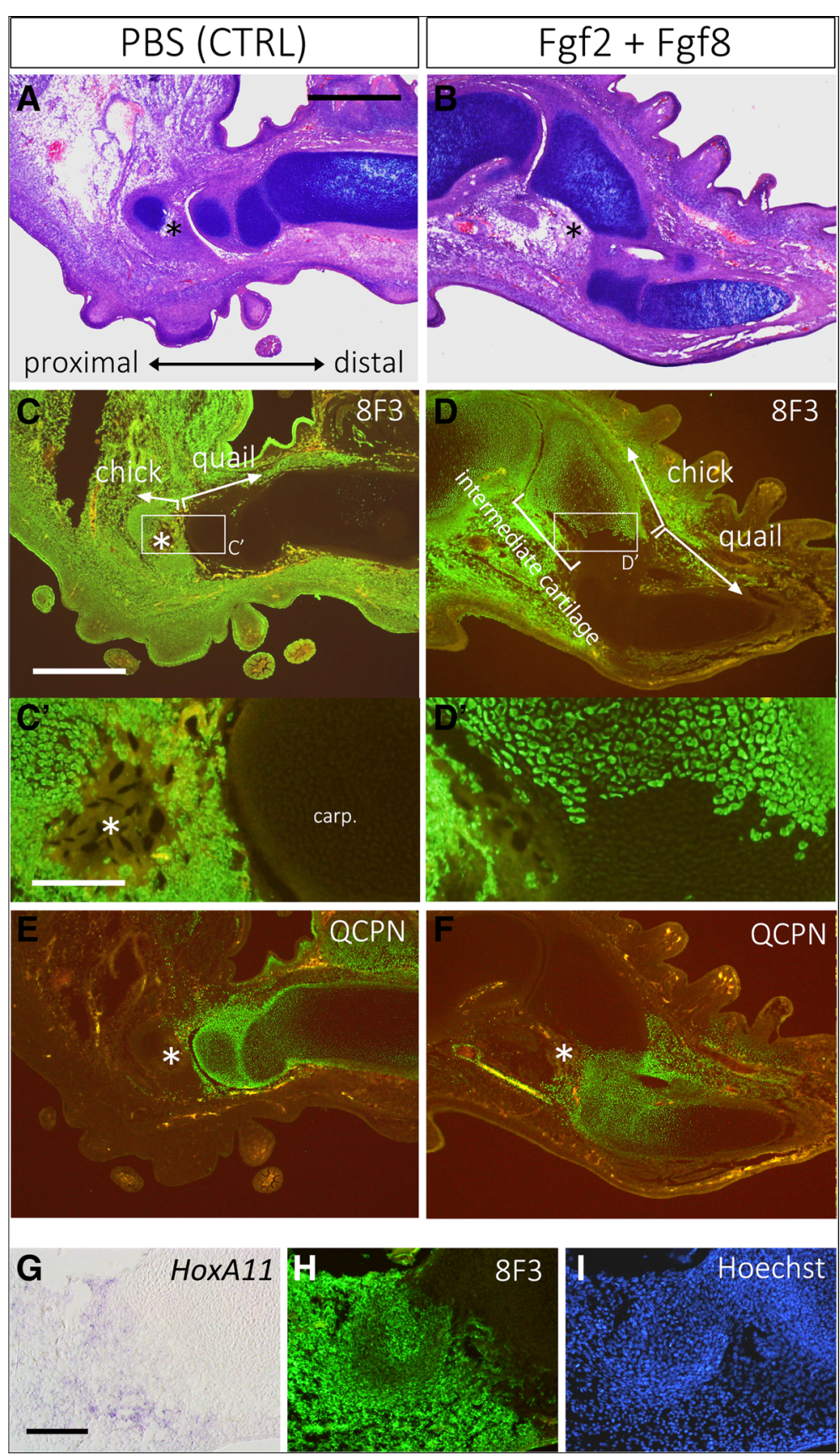

Fig. 7 Cellular contribution of the intercalary induced cartilage in chick limb buds. The grafted hand part was derived from a quail limb bud. a, $\mathbf{c}$, e The control sample. $\mathbf{b}, \mathbf{d}$, f f Fgf 2 + Fgf8 bead-grafted limb. a, b Histological observation. (c-f) Immunofluorescent analysis using the antibody specific for chick cells ( $8 \mathrm{~F} 3 ; \mathbf{c}, \mathbf{d})$ and for quail cells (QCPN; $\mathbf{e}, \mathbf{f})$. $\mathrm{C}^{\prime}$ and $\mathrm{D}^{\prime}$ are the higher magnification views of the boxed regions in $\mathbf{c}$ and $\mathbf{d}$, respectively. Asterisks indicate the beads. Carp. $=$ carpal cartilage. $(G-l)$ The relationship between the HoxA11 expression domain and the 8 F3 expression domain was investigated in the adjacent sections. $\mathbf{g}$ HoxA11 expression. $\mathbf{h} 8 \mathrm{~F} 3$ antigen expression on the adjacent section. I is Hoechst staining for nuclei and is the identical section to $H$. The scale bars in $A, C, C^{\prime}$, and $G$ are $500,500,100$, and $100 \mu \mathrm{m}$, respectively

involved in a zeugopodial part. TUNEL analysis revealed that Fgf2 + Fgf8-soaked bead grafting suppressed apoptosis $16 \mathrm{~h}$ after the surgery (Fig. 5c). It has already been demonstrated that Fgf signaling has the ability to suppress cell death in chicken limb buds $[46,47]$. This apoptosis pattern implies that contaminated zeugopodial cells were eliminated without exogenous Fgf application, and that exogenous Fgf application allowed these populations to survive. We cannot dismiss this possibility completely. Cell lineage analysis, however, revealed that most of the induced 
zeugopodial elements were derived from the proximal stump tissues (Fig. 7). Thus, even if zeugopodial cell contamination were present, its contribution to the intercalary induced structure must not be great. As for the proximal part (the stump), the skeletal pattern obtained in the grafting experiments (Fig. 2c, d, Additional file 1: Figure S1) indicates that no zeugopodial elements are contained. Many samples showed that the induced zeugopodial elements directly extended from the proximal half of the stylopod (Additional file 1: Figure S1). This skeletal pattern strongly suggests that the amputation was correctly performed at the middle of the stylopod, at least in those samples. Given these findings, it is evident that chick limb buds, like urodele amphibians, have the ability to induce the intercalary response.

\section{Proximal-dominant cellular contribution to induced cartilage}

Classical observation showed that intercalary induced tissues were only derived from proximal tissues [36-38]. The present study may demand a small modification of this insight. The labeling technology employed in classical studies was not as accurate compared to that of presentday technology [36-38, 48]. The present study used a GFP transgenic animal to trace cell lineage. Furthermore, a hand graft was taken instead of a blastema graft in the present manuscript. Compared to a blastema, a mature hand was obvious about an amputation site since carpal cartilages instruct a guaranteed amputation site. Because of this, the elimination of zeugopodial elements in the hand grafting experiment was certain. Therefore, it is thought that the combination of the GFP transgenic animal and the hand grafting surgery resulted in a more reliable experimental design. Consistent with the results of classical experiments, it was clearly demonstrated that proximal tissues are the major contributor of intercalary induced tissues (Fig. 6). Detailed cell lineage analysis also revealed that the distal part of the intercalary induced tissues was derived from the distal tissues (Fig. $6 \mathrm{~g}-\mathrm{s}$ ). This distal cell contribution to proximal regenerating tissues was already predicted in our previous studies [7, 49]. Similar cellular contribution in an intercalary response was reported in a planarian study [50]. If a small piece of head piece is grafted into a tail piece, an intercalary response can be induced that results in restoration of the pharyngeal region (intermediate). The induced structure consists mainly of host cells from the tail piece although there are some from the head piece. Given this, coordinated cellular contribution is likely a conserved mechanism of the intercalary response from invertebrates to vertebrates.

Cell lineage tracing analysis in chick intercalary responses revealed the proximal-dominant contribution to intercalary induced tissues (Fig. 7). This is consistent with the axolotl results (Fig. 6). The distal part of the intercalary induced zeugopod was derived from the distal graft although the proximal part was derived from the proximal stump tissues (Fig. 7d, f). Importantly, HoxA11 expression was re-induced in the stump (Fig. $7 \mathrm{~g}-\mathrm{i})$. Generally, proximal parts consist of much more differentiated and determined cells compared to distal parts. Whether or not determined stylopod cells are reprogrammed into zeugopodial cells remains to be investigated. Given the reprogramming of the stylopod tissues, the application of an Fgf2 + Fgf8 bead may play a part. Application of ectopic Fgf2 + Fgf8 in a skin wound is sufficient to induce a blastema in urodele amphibians [18]. Furthermore, blastema induction by Fgf signaling can be observed in some species $[17,51]$. In addition, blastemas always possess distal identities [52]. Fgf2 + Fgf8 application can actually induce a blastema with distal information from a proximal structure. Similarly, Fgf2 + Fgf8 may induce cells with a distal identity from proximal tissues in chick limb buds. Although the detailed mechanisms remain largely unknown, it is very likely that cells in the proximal region are transformed into relatively distal cells. This process to generate distal cells from proximal cells may be called "regeneration" since limb regeneration basically involves the generation of distal structures from remaining proximal stump tissues.

\section{Conserved molecular mechanism of the intercalary response}

Fgf signaling is the key molecular signaling that regulates the intercalary response. This was first speculated by Shimizu-Nishikawa et al. in Xenopus limb development [22]. Basically, Хепориs limb buds lack intercalary ability. The ectopic application of Fgf8, however, induced intercalary responses [22]. The involvement of Fgf signaling in the intercalary response in a urodele amphibian was subsequently reported [21]. Fgf2 application between a hand and a stylopod could induce the zeugopodial elements [21]. This observation was confirmed in the present study (Fig. 6). Even in mice, Fgf signaling regulates intercalary limb developmental mechanisms [20]. Multiple knockout of Fgf genes resulted in a loss of intermediate skeletal elements although the autopod and stylopod were relatively normal. In the case of invertebrates, intercalary regeneration is well known in planarian regeneration [1]. It is still uncertain if Fgf signaling mediates the intercalary regeneration in planarians. An Fgf receptor-like gene, nou-darake, was, however, reported in a planarian [53]. Knockdown of nou-darake resulted in misformation of the proximodistal structure. Furthermore, it was reported that mitogen-activated protein kinase (MAPK)/extracellular signal-related kinase (ERK) signaling is essential for intercalary planarian 
regeneration [54]. ERK is well recognized as one of the major targets of Fgf signaling [55]. Disruption of ERK signaling impairs planarian regeneration. Thus, it is likely that an Fgf-mediated intercalary mechanism plays a role in both limb morphogenesis and in more general morphogenesis.

The present study shows that the Fgf signalingdependent intercalary response can be withdrawn in a chick limb bud. It is reasonable to consider that chick limb development is regulated in a similar manner as mouse limb development. Integrity between the present study and classic studies is found when we focus on the Fgf expression domain in a chick limb bud. Fgf genes are expressed in the apical ectodermal ridge (AER), which is located at the very distal end of a developing limb bud $[56,57]$. A narrow distal domain in a limb bud mesenchyme expresses Fgf10 to maintain Fgf genes in the AER. This suggests that the expected zeugopodial region is Fgf-free as the developmental stages progress. In the very early stages, limb buds are very proximodistally thin. Therefore, Fgfs from the AER and mesenchyme may be able to reach a proximal domain. This may explain the intercalary response in the early stages of chick limb buds [41, 42]. In later stages, limb buds become longer. A distal graft cannot provide sufficient Fgfs to proximal tissues from the distal tip because of its own width. This may explain why the intercalary response could not be observed in relatively later stages. This hypothesis fits with the previous studies that demonstrated short range signal from the AER induces the intercalary response in early chicken embryo [58-61]. Interestingly, Fgf8, which is expressed in the AER in chick limb buds, is expressed in axolotl limb blastema "mesenchyme" [62, 63]. Furthermore, Xenopus Fgf8 is expressed in a blastema epithelium [64]. Application of an ectopic Fgf8 into a Xenopus limb bud mesenchyme causes intercalary regulation [22]. This appears to be a reasonable relationship between an $F g f$ expression domain and an intercalary ability. Evolutionary transition of AER expressing an Fgf gene expression domain would account for the evolutionary loss of the intercalary regeneration system. Regardless, chick limb bud cells continue to retain reactivity to Fgfs, leading to the intercalary response.

\section{Regeneration in amniotes}

The present results can be regarded as regeneration of intermediate structures. To date, the induction of a regeneration blastema, which is a structure with a distal positional identity, remains a future challenge that needs to be solved in amniotes. There are many cases, however, in which "distal tissues" can be obtained. For example, when a serious tumor grows on a lower arm and demands an amputation, a doctor might obtain a hand part from the abscised limb. It may then be feasible to use the hand part with Fgf application to restore the lost lower arm tissues. Alternatively, extracellular matrix (ECM) from distal tissues might be utilized instead of the hand graft. Recently, it was shown that ECM carries positional information. We may use these distal tissues to induce an intercalary regeneration response.

We still do not how to induce a blastema in amniotes. The organ-level regeneration inducers in amphibians have already been reported, however [51]. Given the conservation of the Fgf signaling-based intercalary mechanism from amphibians to amniotes, it is not surprising that the same inducers play a role in blastema induction in amniotes. Urodele amphibians may not be such unique organisms, and we may be on the way to a better understanding of how to mimic some of their special abilities.

\section{Conclusion}

Generally, amniotes do not have organ-level regeneration ability. This may be attributable to loss of a genetic mechanism regulating regeneration ability. In the present study, we demonstrated that the intercalary responses, which are used in limb regeneration in regeneration competent animals, could be induced by the exogenous Fgf2 and Fgf8 application in chicken limb buds. From this point of view, amniotes, at least chick embryos, may maintain a part of the regeneration mechanism. All of our experiments were performed in the embryonic period. Although the reason why the intercalary responses cannot be seen in a postembryonic body remains unknown, our findings imply that the common organ-level regeneration inducers can induce regeneration responses in amniotes.

Intercalation in regeneration involves interaction of cells having different positional identities. When cells recognize each other as non-neighbor cells, those cells are generating intermediate cells until they acquire neighbor cells. Fgf-signaling influences on cells to recognize and distinguish neighbor cells in the chick limb buds and axolotl limbs. Positional identity has remained an unresolved issue in biology. The results of the present study may contribute to the understanding of positional identities and recognition in limb development and regeneration.

\section{Additional files}

Additional file 1: Figure S1. Skeletal pattern of the intercalary regenerated chick wing by Fgf2 + Fgf8 bead grafting. Skeletal pattern was visualized by Alcian blue staining. The scale bar is $2 \mathrm{~mm}$. (JPEG $1535 \mathrm{~kb}$ )

Additional file 2: Figure S2. Joint-like formation between the host stylopod and the induced zeugopod by Fgf2 + Fgf8 application. (A) Gdf5 expression. (B) Type /l collagen expression. The scale bar is $2 \mathrm{~mm}$. (JPEG $2568 \mathrm{~kb}$ ) 


\section{Acknowledgements}

We are grateful to Drs. N. Wada, T. Amano, D. Saito, and T. Suzuki for constructive comments and advice regarding the experiments. We also thank Ms. T. Satoh for her support with this study.

\section{Funding}

This work was supported by a Grant-in-Aid for Scientific Research (B) \#17H03685, Grant-in-Aid for Challenging Exploratory Research \#17 K19400, and the Japan Agency for Medical Research and Development (AMED).

\section{Availability of data and materials}

The datasets supporting the conclusions of this article are included within the article and in the supplemental figure.

\section{Authors' contributions}

A.M and A.S. performed all experiments. A.S. designed the investigation and wrote the manuscript. Both authors read and approved the final manuscript.

\section{Competing interests}

The authors declare that they have no competing interests.

\section{Publisher's Note}

Springer Nature remains neutral with regard to jurisdictional claims in published maps and institutional affiliations.

\section{Received: 9 October 2017 Accepted: 3 April 2018 Published online: 19 April 2018}

\section{References}

1. Agata K, Tanaka T, Kobayashi C, Kato K, Saitoh Y. Intercalary regeneration in planarians. Dev Dyn. 2003;226:308-16

2. Bando $T$, Hamada $Y$, Kurita $K$, Nakamura T, Mito T, Ohuchi H, Noji S. Lowfat, a mammalian Lix1 homologue, regulates leg size and growth under the Dachsous/fat signaling pathway during tissue regeneration. Dev Dyn. 2011;240:1440-53.

3. Boilly B, Lheureux E, Boilly-Marer Y, Bart A. Cell interactions and regeneration control. Int J Dev Biol. 1990;34:219-31.

4. French V, Bryant PJ, Bryant SV. Pattern regulation in epimorphic fields. Science. 1976;193:969-81.

5. Nakamura T, Mito T, Tanaka Y, Bando T, Ohuchi H, Noji S. Involvement of canonical Wnt/wingless signaling in the determination of the positional values within the leg segment of the cricket Gryllus bimaculatus. Develop Growth Differ. 2007:49:79-88.

6. Gardiner DM, Endo T, Bryant SV. The molecular basis of amphibian limb regeneration: integrating the old with the new. Semin Cell Dev Biol. 2002;13:345-52

7. Makanae A, Mitogawa K, Satoh A. Implication of two different regeneration systems in limb regeneration. Regen. 2014;1:1-9.

8. Wallace H. Vertebrate limb regeneration. New York: Wiley; 1981.

9. Han M, Yang X, Taylor G, Burdsal CA, Anderson RA, Muneoka K. Limb regeneration in higher vertebrates: developing a roadmap. Anat Rec $B$ New Anat. 2005:287:14-24.

10. Muneoka $\mathrm{K}$, Allan $\mathrm{CH}$, Yang $\mathrm{X}$, Lee J, Han M. Mammalian regeneration and regenerative medicine. Birth Defects Res C Embryo Today. 2008:84:265-80.

11. Simkin J, Sammarco MC, Dawson LA, Schanes PP, Yu L, Muneoka K. The mammalian blastema: regeneration at our fingertips. Regeneration. 2015;2:93-105

12. Yu L, Han M, Yan M, Lee EC, Lee J, Muneoka K. BMP signaling induces digit regeneration in neonatal mice. Development. 2010;137:551-9.

13. Niswander L, Tickle C, Vogel A, Booth I, Martin GR. FGF-4 replaces the apical ectodermal ridge and directs outgrowth and patterning of the limb. Cell. 1993;75:579-87.

14. Summerbell D, Lewis $\mathrm{JH}$, Wolpert L. Positional information in chick limb morphogenesis. Nature. 1973;244:492-6.

15. Kostakopoulou K, Vogel A, Brickell P, Tickle C. 'Regeneration' of wing bud stumps of chick embryos and reactivation of Msx-1 and Shh expression in response to FGF-4 and ridge signals. Mech Dev. 1996: 55:119-31.

16. Taylor GP, Anderson R, Reginelli AD, Muneoka K. FGF-2 induces regeneration of the chick limb bud. Dev Biol. 1994;163:282-4.
17. Makanae A, Mitogawa K, Satoh A. Cooperative inputs of bmp and Fgf signaling induce tail regeneration in urodele amphibians. Dev Biol. 2016;410:45-55

18. Satoh A, makanae A, Hirata A, Satou Y. Blastema induction in aneurogenic state and Prrx-1 regulation by MMPs and FGFs in Ambystoma mexicanum limb regeneration. Dev Biol. 2011;355:263-74.

19. Yokoyama $H$, Ide $H$, Tamura K. FGF-10 stimulates limb regeneration ability in Xenopus laevis. Dev Biol. 2001;233:72-9.

20. Mariani FV, Ahn CP, Martin GR. Genetic evidence that FGFs have an instructive role in limb proximal-distal patterning. Nature. 2008:453:401-5.

21. Satoh A, Cummings GM, Bryant SV, Gardiner DM. Regulation of proximaldistal intercalation during limb regeneration in the axolotl (Ambystoma mexicanum). Develop Growth Differ. 2010b;52:785-98.

22. Shimizu-Nishikawa K, Takahashi J, Nishikawa A. Intercalary and supernumerary regeneration in the limbs of the frog, Xenopus laevis. Dev Dyn. 2003:227:563-72.

23. Hamburger $\mathrm{V}$, Hamilton $\mathrm{HL}$. A series of normal stages in the development of the chick embryo. J Morphol. 1951;88:49-92.

24. Sato K, Koizumi Y, Takahashi M, Kuroiwa A, Tamura K. Specification of cell fate along the proximal-distal axis in the developing chick limb bud. Development. 2007;134:1397-406.

25. Endo T, Gardiner DM, Makanae A, Satoh A. The accessory limb model: an alternative experimental system of limb regeneration. Methods Mol Biol. 2015;1290:101-13.

26. Mitogawa K, Makanae A, Satoh A, Satoh A. Comparative analysis of cartilage marker gene expression patterns during axolotl and Xenopus limb regeneration. PLoS One. 2015;10:e0133375.

27. Makanae A, Hirata A, Honjo Y, Mitogawa K, Satoh A. Nerve independent limb induction in axolotls. Dev Biol. 2013;381:213-26.

28. Satoh A, Makanae A, Wada N. The apical ectodermal ridge (AER) can be reinduced by wounding, wnt-2b, and fgf-10 in the chicken limb bud. Dev Biol. 2010c;342:157-68

29. Wada N, Kawakami Y, Ladher R, Francis-West PH, Nohno T. Involvement of Frzb-1 in mesenchymal condensation and cartilage differentiation in the chick limb bud. Int J Dev Biol. 1999:43:495-500.

30. Capdevila J, Tsukui T, Rodriquez Esteban C, Zappavigna V, Izpisua Belmonte JC. Control of vertebrate limb outgrowth by the proximal factor Meis2 and distal antagonism of BMPs by gremlin. Mol Cell. 1999;4:839-49.

31. Tiecke E, Bangs F, Blaschke R, Farrell ER, Rappold G, Tickle C. Expression of the short stature homeobox gene Shox is restricted by proximal and distal signals in chick limb buds and affects the length of skeletal elements. Dev Biol. 2006:298:585-96.

32. Yokouchi $Y$, Sasaki H, Kuroiwa A. Homeobox gene expression correlated with the bifurcation process of limb cartilage development. Nature. 1991; 353:443-5.

33. Schneider I, Shubin $\mathrm{NH}$. The origin of the tetrapod limb: from expeditions to enhancers. Trends in genetics. 2013;29:419-26.

34. Tamura $\mathrm{K}$, Yonei-Tamura S, Yano $\mathrm{T}$, Yokoyama $\mathrm{H}$, Ide $\mathrm{H}$. The autopod: its formation during limb development. Dev, Growth Differ. 2008;50 Suppl 1: S177-87.

35. Wellik DM, Capecchi MR. Hox10 and Hox11 genes are required to globally pattern the mammalian skeleton. Science. 2003:301:363-7.

36. Iten LE, Bryant SV. The interaction between the blastema and stump in the establishment of the anterior-posterior and proximal-distal organization of the limb regenerate. Dev Biol. 1975;44:119-47.

37. Pescitelli MJ Jr, Stocum DL. The origin of skeletal structures during intercalary regeneration of larval Ambystoma limbs. Dev Biol. 1980;79:255-75.

38. Stocum DL. Regulation after proximal or distal transposition of limb regeneration blastemas and determination of the proximal boundary of the regenerate. Dev Biol. 1975;45:112-36.

39. Bryant SV, Iten LE. Intercalary and supernumerary regeneration in regenerating the mature limbs of Notophthalmus viridescens. J Exp Zool. 1977:202:1-16.

40. Towers M, Tickle C. Growing models of vertebrate limb development. Development. 2009;136:179-90.

41. Kieny M, Pautou MP. Proximo-distal pattern regulation in deficient avian limb buds. Wilhelm Roux's Arch Dev Biol. 1977:183:177-91.

42. Summerbell D. Regulation of the deficiencies along the proximal distal axis of the chick wing-bud: a quantitative analysis. J Embryol Exp Morphol. 1977;41:137-59. 
43. Healy C, Uwanogho D, Sharpe PT. Regulation and role of Sox9 in cartilage formation. Dev Dyn. 1999;215:69-78.

44. Swalla BJ, Upholt WB, Solursh M. Analysis of type II collagen RNA localization in chick wing buds by in situ hybridization. Dev Biol. 1988; 125:51-8.

45. Scotti, M., Kherdjemil, Y., Roux, M., Kmita, M., 2015. A Hoxa13:Cre mouse strain for conditional gene manipulation in developing limb, hindgut, and urogenital system. Genesis (New York, N.Y. : 2000) 53, 366-376.

46. Buckland RA, Collinson JM, Graham E, Davidson DR, Hill RE. Antagonistic effects of FGF4 on BMP induction of apoptosis and chondrogenesis in the chick limb bud. Mech Dev. 1998:71:143-50.

47. Pajni-Underwood S, Wilson CP, Elder C, Mishina Y, Lewandoski M. BMP signals control limb bud interdigital programmed cell death by regulating FGF signaling. Development. 2007;134:2359-68.

48. Stocum DL. Regenerative Biol Med. 2nd ed: Elsevier (Academic Press); 2012. https://www.elsevier.com/books/regenerative-biology-and-medicine/ stocum/978-0-12-384860-4.

49. Satoh A, Cummings GM, Bryant SV, Gardiner DM. Neurotrophic regulation of fibroblast dedifferentiation during limb skeletal regeneration in the axolotl (Ambystoma mexicanum). Dev Biol. 2010a;337:444-57.

50. Kobayashi C, Nogi T, Watanabe K, Agata K. Ectopic pharynxes arise by regional reorganization after anterior/posterior chimera in planarians. Mech Dev. 1999;89:25-34.

51. Satoh A, Mitogawa K, Makanae A. Regeneration inducers in limb regeneration. Develop Growth Differ. 2015;57:421-9.

52. Satoh A, Gardiner DM, Bryant SV, Endo T. Nerve-induced ectopic limb blastemas in the axolotl are equivalent to amputation-induced blastemas. Dev Biol. 2007:312:231-44.

53. Cebria F, Kobayashi C, Umesono Y, Nakazawa M, Mineta K, Ikeo K, Gojobori T, Itoh M, Taira M, Sanchez Alvarado A, Agata K. FGFR-related gene nou-darake restricts brain tissues to the head region of planarians. Nature. 2002;419:620-4.

54. Umesono Y, Tasaki J, Nishimura Y, Hrouda M, Kawaquchi E, Yazawa S, Nishimura O, Hosoda K, Inoue T, Agata K. The molecular logic for planarian regeneration along the anterior-posterior axis. Nature. 2013;500:73-6.

55. Turner N, Grose R. Fibroblast growth factor signalling: from development to cancer. Nat Rev Cancer. 2010:10:116-29.

56. Benazet JD, Zeller R. Vertebrate limb development: moving from classical morphogen gradients to an integrated 4-dimensional patterning system. Cold Spring Harb Perspect Biol. 2009;1:a001339.

57. Fernandez-Teran M, Ros MA. The apical ectodermal ridge: morphological aspects and signaling pathways. Int J Dev Biol. 2008;52:857-71.

58. Amprino R, Camosso M. On the role of the "apical ridge" in the development of the chick embryo limb bud. Acta Anat. 1959:38:280-8.

59. Kieny M. Étude du mécanisme de la régulation dans le développement du bourgeon de membre de l'embryon de poulet. I. Réqulation des excédents. Dev Biol. 1964;9:197-229.

60. Rosello-Diez A, Torres M. Regulative patterning in limb bud transplants is induced by distalizing activity of apical ectodermal ridge signals on host limb cells. Dev Dyn. 2011;240:1203-11.

61. Summerbell D. Evidence for regulation of growth, size and pattern in the developing chick limb bud. J Embryol Exp Morphol. 1981;65(Suppl):129-50.

62. Han MJ, An JY, Kim WS. Expression patterns of Fgf-8 during development and limb regeneration of the axolotl. Dev Dyn. 2001;220:40-8.

63. Nacu E, Gromberg E, Oliveira CR, Drechsel D, Tanaka EM. FGF8 and SHH substitute for anterior-posterior tissue interactions to induce limb regeneration. Nature. 2016;533:407-10.

64. Endo T, Tamura K, Ide H. Analysis of gene expressions during Xenopus forelimb regeneration. Dev Biol. 2000;220:296-306.

Ready to submit your research? Choose BMC and benefit from:

- fast, convenient online submission

- thorough peer review by experienced researchers in your field

- rapid publication on acceptance

- support for research data, including large and complex data types

- gold Open Access which fosters wider collaboration and increased citations

- maximum visibility for your research: over $100 \mathrm{M}$ website views per year

At BMC, research is always in progress.

Learn more biomedcentral.com/submissions 